\title{
Disparities and Racism Experienced Among Older African Americans Nearing End of Life
}

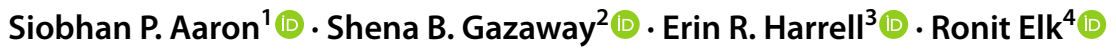 \\ Accepted: 2 September 2021 / Published online: 14 December 2021 \\ (c) The Author(s), under exclusive licence to Springer Science+Business Media, LLC, part of Springer Nature 2021
}

\begin{abstract}
Purpose of Review The purpose of this review is to examine racism in healthcare as it relates to older African American adults. We focus on health disparities in old age and medical mismanagement throughout their lifespan.

Recent Findings In the United States there have been extensive medical advances over the past several decades. Individuals are living longer, and illnesses that were deemed terminal in the past are now considered chronic illnesses. While most individuals living with chronic illness have experienced better quality of life, this is not the case for many African American older adults.

Summary Older African American adults are less likely to have their chronic illness sufficiently managed and are more likely to die from chronic illnesses that are well controlled in Whites. African American older adults also continue to suffer from poorer healthcare outcomes throughout the lifespan to end-of-life.
\end{abstract}

Keywords African American · Chronic illness · End of life care · Health inequality

In the United States overall people are living longer, yet African Americans aged 18-49 are twice as likely to die from heart disease than Whites [1]. This disparity is in part due to younger African Americans being diagnosed with chronic diseases normally seen in older populations [1]. Having lived with chronic diseases longer, African Americans experience significant symptom burden and higher risk of complications as they age in comparison to their nonHispanic White counterparts [1, 2]. This increased morbidity related to living longer with chronic illness can intensify the suffering older African American patients experience towards the end-of-life (EOL) [3].

This article is part of the Topical Collection on Palliative Care

Siobhan P. Aaron

Siobhan.aaron@utah.edu

1 College of Nursing, University of Utah, 102000 E, Salt Lake City, UT 84112, U.S.A.

2 School of Nursing, University of Alabama Birmingham, Birmingham, AL, U.S.A.

3 Department of Psychology, University of Alabama, Tuscaloosa, AL, U.S.A.

4 Department of Medicine, University of Alabama Birmingham, Birmingham, AL, U.S.A.
The emergence of COVID-19 in early 2020 placed further emphasis on the reprehensible mortality gap between African Americans and Whites as report after report demonstrated Blacks accounted for the highest mortality rate - a rate that was 2.3 times higher than Whites and Asian Americans [4]. These numbers support the notion that the Black Community is facing a syndemic (two concurrent epidemics, in this case COVID-19 and racism). Syndemic theory explains the tendency for multiple co-existing and interacting epidemics to develop under conditions of health and social disparities [5, 6]. With increased COVID-19 exposure and mortality rates intersecting with persistent national health and social inequalities, generated by systemic racism, it is evident that Black Americans live in various degrees of crisis as documented by former Surgeon General, Dr. David Satcher, in a landmark paper [7].

Due to current social, economic, and political structures, many African Americans experience chronic illness within an impoverished environment with decreased access to quality primary care and professional medical support [8]. The purpose of this review is to explore racism in healthcare as it relates to older African American adults' experience with health disparities and medical mismanagement throughout their lifespan. We will highlight how these detrimental 
experiences influence the disparities seen in advanced care planning and EOL service utilization, in turn impacting African Americans' lived experience with serious illness until death. We conclude this article by sharing recommendations to address these concerns, primarily through African American community involvement in the development of culturally responsive programs geared to drive clinician conversations with seriously ill African Americans and their families.

\section{Disparities and the Chronic Illness Experience}

\section{Pain Mismanagement}

Older African American adults often report experiencing pain levels of five and higher, as well as higher levels of insomnia, depression, and inferior memory quality [9]. Yet, older African American adults are at increased risk of being undertreated for pain. In addition to having their pain underaccessed, they are less likely to receive opioid and nonopioid-based medications [10-12]. A study analyzing data from a dataset within the Baltimore Study of Black Aging series, assessed activities of daily living (ADL) and pain in older African American adults [13]. Results showed that bodily pain and two or more comorbidities were significantly associated with ADL disability. Another study looking at a subset of the Health and Retirement Study (HRS) data in $2010(n=1,925)$ showed that older African American female adults over 65 reported the most chronic pain and higher pain intensity outcomes [14]. Study results showed that over one-third of Blacks in this study described that pain affected their ability to meet their financial needs. A meta-analysis [15] that examined racial differences in osteoarthritis pain between African American/Blacks and Non-Hispanic Whites found higher pain severity in African Americans, and another large study that included 4707 cancer survivors, affirmed that race/ethnicity remain a significant barrier to pain [16]. Pain negatively affects individuals psychologically, physiologically, cognitively, spiritually, and physically, yet pain in older African American adults continues to be undermanaged [12]. This results not only in increased suffering, but also strains relationships with providers leading to distrust of clinicians and the healthcare system [17].

These statistics support the notion that due to the subjective nature of pain, implicit bias in clinicians as well as other negative perceptions of African Americans, including racism, can lead to undertreatment $[18,19]$. Prevalent misconceptions among clinicians include the historical misconception that African Americans have a high pain tolerance and do not experience pain sensitivity in the same way that
Whites do [20]. Another frequently cited mistaken belief by clinicians is that African American patients in pain are drug seeking, resulting in providers not assessing the pain and also not treating it [21].

Over and above clinicians' biased misconceptions, are misconceptions perpetuated by researchers who create pain assessment measures. Many pain scales and measures have not been tested for validity and reliability in diverse populations [11]. Not using valid tools in diverse populations can be a crucial contributor to pain being under-assessed and undertreated. All patients deserve the right to have their pain optimally managed. Yet African American patients will continue to be under-assessed and undertreated as long as there is a reluctance by clinicians (and by the healthcare system to hold clinicians accountable) to assess-and adequately treat-these patients for pain.

\section{Poor Communication and Disregard of Cultural, Spiritual and Religious Values of African Americans}

A second area in which African Americans experience poorer provision of healthcare is with inferior provider communication, information giving, patient participation, and participatory decision-making compared to White patients [22]. In studies of African American older adults, poorer patient-provider communication was seen when the patient belonged to a racial/ethnic minority group, and if the clinician possessed decreased cultural competence and unconscious bias [23, 24].

The structural influence model of health communication posits that EOL discussions lead to communication goals (including terminal illness awareness, treatment preferences, and do-not-resuscitate (DNR) codes). Social factors greatly influence how EOL conversations go and serve to predict subsequent clinical outcomes. This has been supported by research [25], that found that Black patients who engaged in EOL discussions with their physician were more likely to prefer symptom-directed care than those who did not. Having a DNR order in place neither decreased the likelihood that Black patients would use hospice care in their last week of life nor increased the likelihood that Black patients received EOL care consistent with their preferences.

For many African Americans, EOL care is influenced by religion and spirituality. The Pew Report found that $80 \%$ of African Americans are religious [26]. Thus, many African Americans rely on their faith when reflecting on mortality [27]. This faith has sustained African Americans during tumultuous moments from slavery to segregation to currentday events resulting from systematic racism. Thus, spirituality is vital in African American Christian patients' and 
families' lives; and, when they face EOL, they pull from their faith and speak of "miracles" and healing [28].

At end of life, the objective is to provide "goal-concordant" care based on what the patient and family value and want. Despite care that is not goal-concordant being considered as a "medical error" [29], the hope for a miracle and the belief that God is the ultimate decider of who lives and who dies is frequently dismissed and disregarded [27, 29-31]. African American patients and families deserve care that is equitable and preserves the life that their loved one has lived and acknowledges their faith and beliefs [32]. Yet, studies have shown that African Americans have a higher rate of "nongoal-concordant care" than Whites [33].

In summary, despite communication between Black patients and their physician/healthcare providers, health outcome disparities persist. These disparities suggest that there is a deficiency, disregard, and misperception by clinicians. When it comes to concordant care, the goal is not just to have conversations, but to understand and respect the patient and their family's wishes.

\section{Advance Care Planning}

We have presented strong evidence that African Americans are at high risk of suffering disproportionately from substandard, and at times biased, care throughout their serious illness experience. This inferior care results in African Americans being less likely to utilize EOL services compared to Whites [34-37]. Among African Americans, advance care planning (ACP) completion rates are substantially lower [29, 37, 38]. African Americans are more likely to pursue informal EOL planning. Yet even when African Americans have their preferences recorded, they are less likely than Whites to have their preferences upheld by clinicians in hospitals [25].

In a systematic review of the literature focusing on barriers to completing ACP in the African American community, [29] several themes emerged including beliefs about illness, death and dying and suffering; family and community structure; support and burden; religion and spirituality; preferences regarding life-sustaining treatment; health literacy; style of processing and enacting health decisions; communication with medical teams; and attitudes about EOL planning. Based on these findings, the conclusion was that a combination of personal, interpersonal, and systemlevel factors, including the strong influence of slavery in the US, has shaped African Americans' beliefs and attitudes about EOL.

In a meta-analysis [39], De Souza et al. found that older African American adults are less likely than their same aged counterparts to complete advance directives. Reasons for this included the avoidance of EOL conversations, miscommunication between providers and patient/caregivers, differing cultural beliefs, and lack of trust for healthcare providers $[39,40]$. Since clinicians often rely on the Advance Directive document to guide EOL care, in the absence of such documentation African Americans are less likely to have their treatment preferences observed. Yet a provider engaging in a conversation with the patient and family, in which the patient's wishes and values are elicited, could easily result in a clear understanding. The key is both having the conversation and respecting the cultural, religious, and spiritual values that originate from the conversation.

\section{Hospice and Palliative Care}

African Americans often opt for more intensive treatment options at the EOL. A study with a cohort of 1212 individuals from the Reasons for Geographic and Racial Difference in Stroke (REGARDS 2003-2007) dataset showed that Blacks were more likely to continue treatment at the EOL and less hospice usage than their White counterparts [41]. For older adults with dementia and the likelihood of live discharge from hospice, two studies found that African Americans weresignificantly more likely to experience a live discharge from hospice than Whites; some of these discharges occurred following acute hospitalizations [42, 43]. These findings signify that African Americans experience arduous disruptions in care at EOL resulting in a higher likelihood of them not being able to benefit from these services due to acute hospitalization [43].

Multiple reasons are cited in the literature for the underuse of hospice services. The medical workforce's demographic composition is a significant factor to discuss. Many African Americans are hesitant to place trust in medical professionals not belonging to the same race; this mistrust is in part due to a long and very painful history of medical segregation, uninformed experimentation, and current exposure to microaggressions and implicit bias [22, 25, 33]. Social-environmental barriers, such as financial burden, care setting or geographic location, and provider communication, have also negatively affected African Americans' access to EOL services [44]. Finally, the care of African Americans by family members ("who else did we have to depend on?") for generations continues today, where the older loved one is kept at home with the family caring for him/her, regardless of the sacrifice required (Fig. 1) [45]. 
Fig. 1 Racism in Healthcare for the African American Older Adult

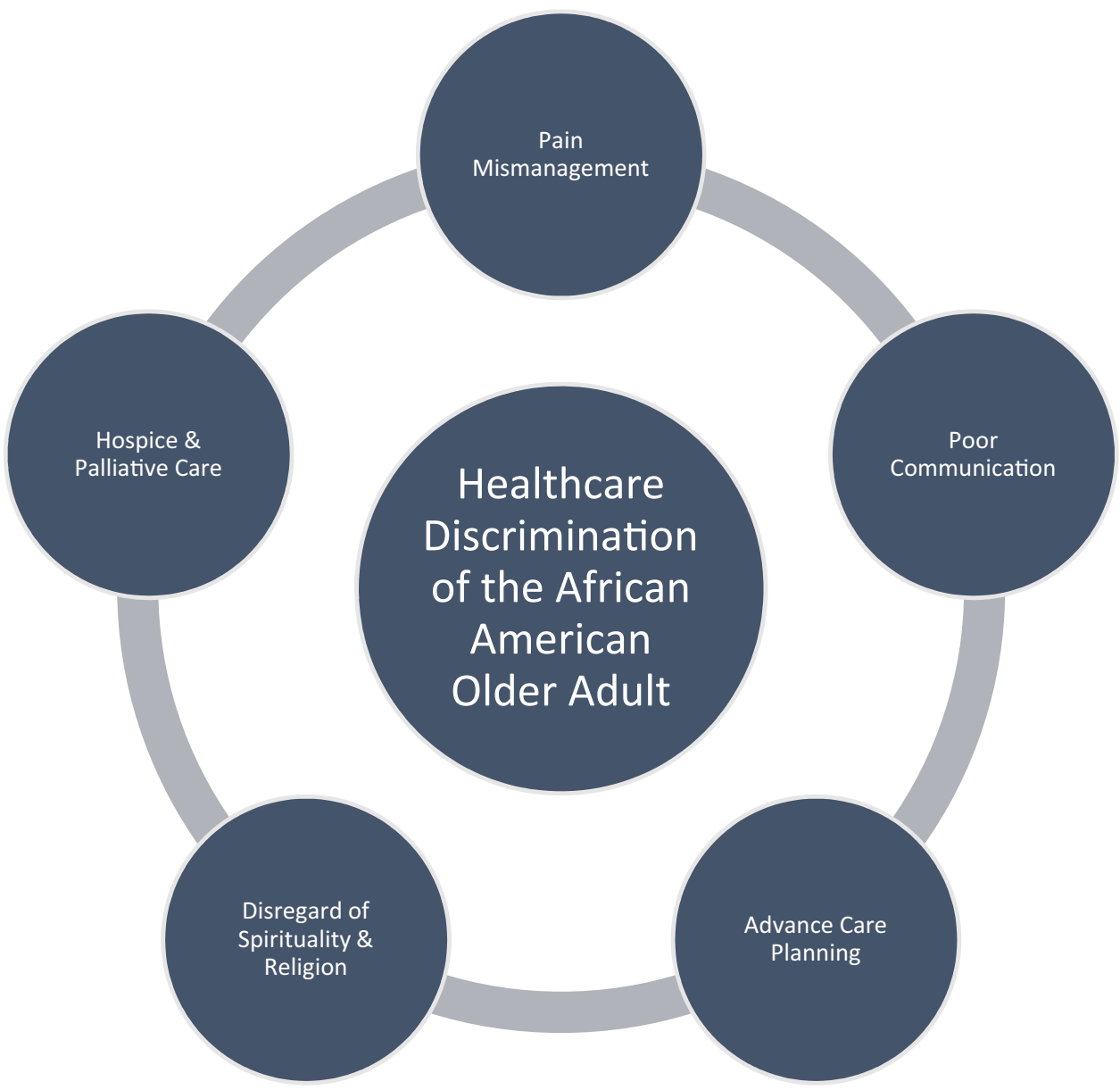

\section{Recommendations}

\section{Increase Culturally Responsive End-of-Life Programs}

African Americans suffer in serious illness and EOL due to a lack of culturally appropriate programs that are designed to address their concerns adequately and equitably. Culture fundamentally shapes how individuals make meaning out of illness, suffering, and dying, [46, 47], and it strongly influences people's responses to diagnosis, illness, and treatment preferences $[48,49]$. Historically EOL care has been rooted in White middle-class cultural and religious values; $[30,50]$, with a different frame of reference, value system, and life experience than most African Americans [51]. Considering patients' and families' cultures is essential in all aspects of palliative care. Palliative care is intended to provide seriously ill older adults with expert care, goals of care discussions, and advance care planning for not only the patient but the caregiver [52]. However, culturally appropriate care models that consider the diverse cultural preferences of seriously ill rural patients and their family caregivers were not available until Elk and team created the first such model, in which community members were the guides for this program, demonstrating its feasibility [45] and currently testing its efficacy [53].

Further efforts are needed to overcome barriers in pain management of African Americans with chronic conditions. One way to do this is to change health care approaches and policies. Recommendations should be based upon metrics that hold organizations accountable for the treatment of pain among all patients. African American patients must have equitable pain assessment and pain treatment [21]. Further research needs to be aimed at using tools in healthcare that capture the voices of minorities.

We also need to extend our efforts to change surface-level content of EOL focused health care education to African American populations. Some studies have attempted this through simple modifications of materials by adding pictures and verbiage that would appeal to African Americans and ensuring that study facilitators represent race and similar participants' social backgrounds [54]. What is missing from these studies is family support and family-centered decision-making, [55] family interconnectedness, and the 
collectivism valued by African Americans [56]. Therefore, significant efforts must be made to gain insight into African American family members' cultural values and serious illness preferences. African Americans often value faith, spiritual beliefs, and spiritual leader guidance [30], especially as they cope with illness and make treatment decisions.

The Black Church is among the most important institutions in the African American community, providing spiritual, social, and health benefits. Pastors are key community leaders; their input and involvement can influence health programs' implementation and outcomes [57]. Acknowledging the cultural perspectives of African American families and pastors who minister to patients is the first step in developing culturally based goals of care interventions. Including these values and spirituality-related elements has the power to improve cognitive, emotional, and behavioral outcomes at EOL and can help overcome multiple barriers to EOL service usage [19, 44, 54].

\section{Honest Communication from Healthcare Team}

Numerous studies have reported the importance of honest/ frank discussion in ACP [58-60]. Ongoing conversations have been shown to be effective in eliciting preferences at EOL [61, 62]. These gradual and culturally respectful conversations have been helpful in prompting conversations about EOL, and in building trust with African American patients and their families Yet a recent study has found that African African healthcare proxies who engaged in EOL discussions with a healthcare provdider report increased unmanaged needs related to sadness and anxiety [63]. Further exploration of this is required, as it seems these conversations are not meeting the full needs of African American healthcare proxies. Provider communication is key to engaging older African Americans and their caregivers in EOL conversations. When racist perceptions or even stereotypical thoughts impact the communication process, there is increased risk for miscommunication and breakdown of the process [42].

Approaches that consider cultural-related preferences should include the healthcare workers' involvement from diverse backgrounds to help create advance directives [64]. A diverse workforce can help guide physicians, patients, and proxies towards an optimal representation of care based on their values, including burden and comfort [65], autonomy and dignity [66], and religiosity/spirituality [67]. A diverse workforce not only attracts diverse patient populations but increases patient satisfaction [68]. Studies have also suggested that interdisciplinary teams work to enhance communications [69]. Interdisciplinary teams have several roles. If one discipline cannot reach the patient there is opportunity that another can. These types of teams collectively hold a breadth of knowledge. Through this combined knowledge, the team can provide patients with relevant and timely information. Furthermore, when multiple disciplines work together, it increases patient satisfaction, decreases patient adverse safety events, and improves quality of care [70].

Next, having culturally concordant discussions may be impactful to patient-provider discussions. This may be most helpful in having discussions about prognosis, as well as having conversations that provoke EOL care preferences. Culturally centered conversations allow individuals to integrate their beliefs and values during the treatment plan. When goals and values are elicited, communication is enhanced [71]. With improved culturally concordant communication, patients and families are more likely to understand prognosis and receive useful information about their diagnosis more readily. By understanding, respecting, and honoring the beliefs and values of African American communities, healthcare providers can cultivate cultural preferences into EOL discussions. Future interventions should be directed at training providers in having discussions that incorporate patient beliefs. Trainings based on African American community recommendations [72] are the key as listening to community voices towards health equity.

With the recent pandemic of COVID-19, the peril that the African American community faces are further showcased. With deaths in this community doubling those of deaths in any other race [4], it is important to address that some African Americans' will lack of trust in the healthcare system. This mistrust in the healthcare system directly contributes to low healthcare utilization, poorer health outcomes, and decreased healthcare literacy [73]. Efforts must be directed towards community outreach and partnerships to raise awareness about the virus and promote health-seeking behaviors. These efforts must be made in a manner that is culturally congruent and incorporates patients' preference.

Research has shown that organizations who train their providers to incorporate patient preferences into their care delivery experience higher patient satisfaction [74]. The healthcare system needs to take ownership of training its professionals to provide care that is accessible to all cultures. A deep reflection of this construct has been done since the murder of George Floyd and the subsequent awakening in the US and beyond [75]. Anti-racism training programs are proliferating, programs that allow providers to examine themselves and engage in deep reflection into their practice in order to combat implicit and systemic bias [76]. Without the healthcare system and clinicians understanding and self-reflecting on concepts taught through such trainings, our society cannot make advances. Anti-racism training is critical for our country to take steps toward an equitable healthcare system. This includes a need for healthcare providers to learn to recognize and respect the patient burden of slavery and racism. The historic mistrust of the healthcare system further multiplies disparate outcomes in healthcare. 


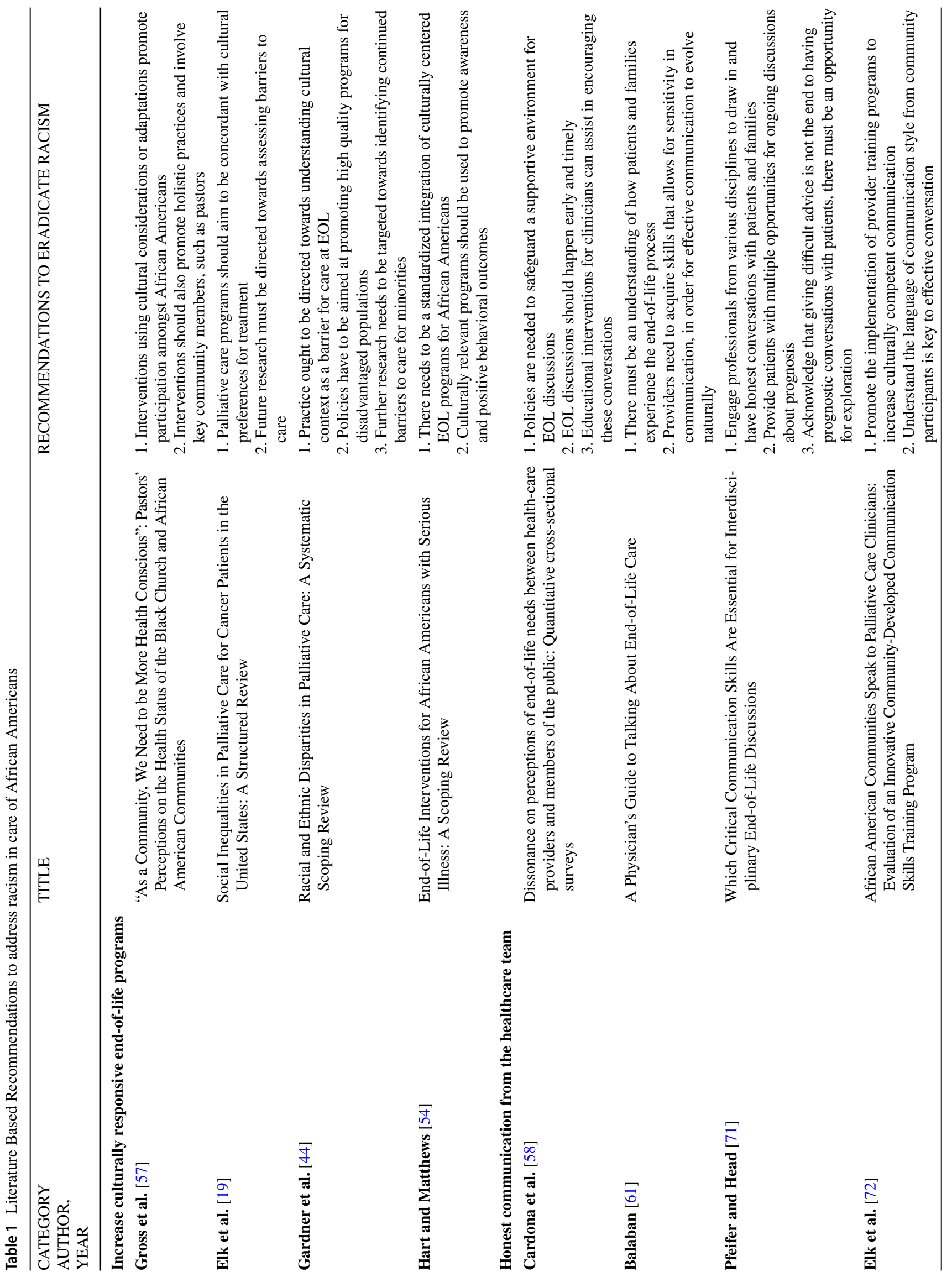




\section{Community Based Participatory Research}

For a fundamental shift in EOL health disparities, African American community voices MUST be listened to and involved in the change [77]. One way to systematically include African American agents in program development and implementation is community-based participatory research [78-80]. CBPR consists of obtaining input from and involvement of members of the local community. Utilizing CBPR methods increases the likelihood that the findings will be accepted and embraced by the community. By way of community-based research, the infusion of different African American perspectives, rural, urban, older adults, and young adults, can be gathered. This understanding of their perspective aims to help improve African Americans' EOL experience drastically [45]. Without these perspectives, any 'culturally' developed program loses its authenticity and value (Table 1).

\section{Conclusion}

There is an unacceptable difference in the way African Americans receive EOL care. It is critical that systemic racism in healthcare be addressed to ensure equitable care. The first step is recognizing, acknowledging, and respecting the inequity, disrespect, and disregard our African American patients have experienced [81]. Next, systemic and individual racism must be addressed at multiple levels: policy level, institutional level; community wide, and individually. Moreover, institutions must partner with communities to build trust between African Americans and the healthcare system. Finally, and most importantly, the voices of the African American community must be heard, there needs to be more representation of African Americans in healthcare, and partnerships between institutions that provide healthcare and the community must be formed if we are to truly achieve health equity for African Americans at EOL [77].

Authors' Contributions All authors served in the development, writing, editing, reviewing, and approval for the submission of this manuscript.

Funding The authors report no funding supported the development of this manuscript.

\section{Compliance with Ethical Standards}

Conflicts of interest/Competing interests The authors have no conflicts or competing interests to disclose.

Human and Animal Rights and Informed Consent This article does not contain any studies with human or animal subjects performed by any of the authors.

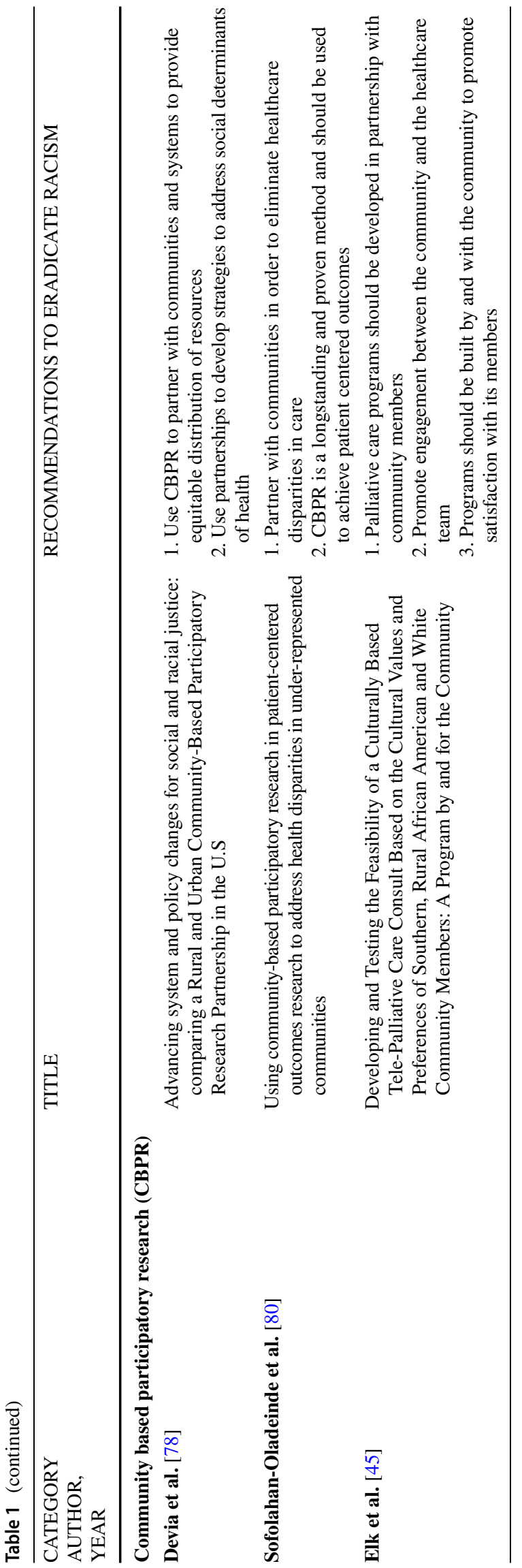




\section{References}

\section{- Of importance}

$\bullet$ Of major importance

1. CDC. African American Health. 2020. https://www.cdc.gov/ media/dpk/healthy-living/african-american-health/index.html. Cited 26 Jun 2021

2. Cunningham TJ. Vital Signs: Racial Disparities in Age-Specific Mortality Among Blacks or African Americans - United States, 1999-2015. 2017. https://www.facebook.com/CDCMM WR. Cited 28 Jun 2021

3. Hendricks Sloan D, Peters T, Johnson KS, Bowie JV, Ting Y, Aslakson R. Church-Based Health Promotion Focused on Advance Care Planning and End-of-Life Care at Black Baptist Churches: A Cross-Sectional Survey. J Palliat Med. 2016;19:190-4.

4. APM RESEARCH LAB STAFF. Color of Coronavirus: COVID19 deaths analyzed by race and ethnicity. 2021. https://www. apmresearchlab.org/covid/deaths-by-race. Cited 26 Jun 2021

5. Poteat T, Millett GA, Nelson LE, Beyrer C. Understanding COVID-19 risks and vulnerabilities among black communities in America: the lethal force of syndemics. Ann Epidemiol. 2020;47:1-3.

6. Bassett MT, et al.The unequal toll of COVID-19 mortality by age in the United States: Quantifying racial/ethnic disparities. 2020. https://www.hsph.harvard.edu/social-and-behavioral-sciences/ 2020/06/23/the-unequal-toll-of-covid-19-mortality-by-agein-the-united-states-quantifying-racial-ethnic-disparities/. Cited 28 Jun 2021

7. Satcher D, Fryer GE, McCann J, Troutman A, Woolf SH, Rust G. What if we were equal? A comparison of the blackwhite mortality gap in 1960 and 2000. Health Aff Proj Hope. 2005;24:459-64

8. Kangovi S, Barg FK, Carter T, Long JA, Shannon R, Grande D. Understanding why patients of low socioeconomic status prefer hospitals over ambulatory care. Health Aff Proj Hope. 2013;32:1196-203.

9. Bazargan M, Yazdanshenas H, Gordon D, Orum G. Pain in Community-Dwelling Elderly African Americans. J Aging Health. 2016;28:403-25.

10. Bhimani RH, Cross LJS, Taylor BC, Meis LA, Fu SS, Allen $\mathrm{KD}$, et al. Taking ACTION to reduce pain: ACTION study rationale, design and protocol of a randomized trial of a proactive telephone-based coaching intervention for chronic musculoskeletal pain among African Americans. BMC Musculoskelet Disord. 2017;18:15.

11. Booker SS, Herr K. The State-of-"Cultural Validity” of SelfReport Pain Assessment Tools in Diverse Older Adults. Pain Med. 2015;16:232-9.

12. Robinson-Lane SG, Booker SQ. Culturally Responsive Pain Management for Black Older Adults. J Gerontol Nurs. 2017;1-8.

13. Walker JL, Thorpe RJ, Harrison TC, Baker TA, Cary M, Szanton SL, et al. The Relationship between Pain, Disability and Gender in African Americans. Pain Manag Nurs Off J Am Soc Pain Manag Nurses. 2016;17:294-301.

14. Janevic MR, McLaughlin SJ, Heapy AA, Thacker C, Piette JD. Racial and Socioeconomic Disparities in Disabling Chronic Pain: Findings From the Health and Retirement Study. J Pain. 2017;18:1459-67.

15. Vaughn IA, Terry EL, Bartley EJ, Schaefer N, Fillingim RB. Racial-Ethnic Differences in Osteoarthritis Pain and Disability: A Meta-Analysis. J Pain Off J Am Pain Soc. 2019;20:629-44.
16. Stein KD, Alcaraz KI, Kamson C, Fallon EA, Smith TG. Sociodemographic inequalities in barriers to cancer pain management: a report from the American Cancer Society's Study of Cancer Survivors-II (SCS-II). Psychooncology. 2016;25:1212-21.

17. Stewart KA, Parshad-Asnani M, Wonkam A, Bollinger J, Ngo Bitoungui V, Wonkam-Tingang E, et al. "Pain is Subjective": A Mixed-Methods Study of Provider Attitudes and Practices Regarding Pain Management in Sickle Cell Disease Across Three Countries. J Pain Symptom Manage. 2021;61:474-87.

18. Burgio KL, Williams BR, Dionne-Odom JN, Redden DT, Noh H, Goode PS, et al. Racial Differences in Processes of Care at End of Life in VA Medical Centers: Planned Secondary Analysis of Data from the BEACON Trial. J Palliat Med. 2016;19:157-63.

19. Elk R, Felder TM, Cayir E, Samuel CA. Social Inequalities in Palliative Care for Cancer Patients in the United States: A Structured Review. Semin Oncol Nurs. 2018;34:303-15.

20. Hoffman KM, Trawalter S, Axt JR, Oliver MN. Racial bias in pain assessment and treatment recommendations, and false beliefs about biological differences between blacks and whites. Proc Natl Acad Sci USA. 2016;113:4296-301.

21. Santoro TN, Santoro JD. Racial Bias in the US Opioid Epidemic: A Review of the History of Systemic Bias and Implications for Care. 2018. https://www.ncbi.nlm.nih.gov/pmc/ articles/PMC6384031/. Cited 6 Jun 2021

22. Shen MJ, Peterson EB, Costas-Muñiz R, Hernandez MH, Jewell ST, Matsoukas K, et al. The Effects of Race and Racial Concordance on Patient-Physician Communication: A Systematic Review of the Literature. J Racial Ethn Health Disparities. 2018;5:117-40.

23. Pérez-Stable EJ, El-Toukhy S. Communicating with diverse patients: How patient and clinician factors affect disparities. Patient Educ Couns. 2018;101:2186-94.

24. Viswanath K, Ramanadhan S, Kontos EZ. Mass Media. In: Galea S, editor. Macrosocial determinants of population health. New York, NY: Springer; 2007. p. 275-94. https://doi.org/10.1007/ 978-0-387-70812-6_13.

25. Mack JW, Paulk ME, Viswanath K, Prigerson HG. Racial Disparities in the Outcomes of Communication on Medical Care Received Near Death. 2010. http://archinte.jamanetwork.com/ article.aspx?doi=10.1001/archinternmed.2010.322. Cited 26 Jun 2021

26. Pew Research Center. 2014 U.S. Religious Landscape Study. 2014. https://www.pewforum.org/dataset/pew-research-center2014-u-s-religious-landscape-study/. Cited 28 Jun 2021

27. Kennard CL. Undying Hope. J Palliat Med. 2016;19:129-30.

28. Moss KO, Douglas SL, Lipson AR, Blackstone E, Williams D, Aaron S, et al. Understanding of Health-related Decision-making Terminology Among Cancer Caregivers. West J Nurs Res. 2021;43:649-59.

29. Sanders JJ, Johnson KS, Cannady K, Paladino J, Ford DW, Block $\mathrm{SD}$, et al. From Barriers to Assets: Rethinking factors impacting advance care planning for African Americans. Palliat Support Care. 2019;17:306-13.

30. Wicher CP, Meeker MA. What influences African American end-of-life preferences? J Health Care Poor Underserved. 2012;23:28-58.

31. Johnson KS, Elbert-Avila KI, Tulsky JA. The influence of spiritual beliefs and practices on the treatment preferences of African Americans: a review of the literature. J Am Geriatr Soc. 2005;53:711-9.

32. Johnson J, Hayden T, True J, Simkin D, Colbert L, Thompson B, et al. The Impact of Faith Beliefs on Perceptions of End-of-Life Care and Decision Making among African American Church Members. J Palliat Med. 2016;19:143-8. 
33. Armstrong K, Putt M, Halbert CH, Grande D, Schwartz JS, Liao K, et al. Prior Experiences of Racial Discrimination and Racial Differences in Health Care System Distrust. Med Care. 2013;51:144-50.

34. Narang AK, Wright AA, Nicholas LH. Trends in Advance Care Planning in Patients With Cancer: Results From a National Longitudinal Survey. JAMA Oncol. 2015;1:601.

35. Lovell A, Yates P. Advance Care Planning in palliative care: A systematic literature review of the contextual factors influencing its uptake 2008-2012. Palliat Med. 2014;28:1026-35.

36. Johnson KS, Payne R, Kuchibhatla MN. What are Hospice Providers in the Carolinas Doing to Reach African Americans in Their Service Area? J Palliat Med. 2016;19:183-9.

37. Johnson KS. Racial and Ethnic Disparities in Palliative Care. J Palliat Med. 2013;16:1329-34.

38. Committee on Approaching Death: Addressing Key End of Life Issues, Institute of Medicine. Dying in America: Improving Quality and Honoring Individual Preferences Near the End of Life. 2015. http://www.ncbi.nlm.nih.gov/books/NBK285681/. Cited 26 Jun 2021

39. De Souza J, Gillett K, Froggatt K, Walshe C. Perspectives of elders and their adult children of Black and minority ethnic heritage on end-of-life conversations: A meta-ethnography. Palliat Med. 2020;34:195-208.

40. Huang IA, Neuhaus JM, Chiong W. Racial and Ethnic Differences in Advance Directive Possession: Role of Demographic Factors, Religious Affiliation, and Personal Health Values in a National Survey of Older Adults. J Palliat Med. 2016;19:149-56.

41. Ornstein KA, Roth DL, Huang J, Levitan EB, Rhodes JD, Fabius CD, et al. Evaluation of Racial Disparities in Hospice Use and End-of-Life Treatment Intensity in the REGARDS Cohort. JAMA Netw Open. 2020;3:e2014639.

42. Luth EA, Russell DJ, Brody AA, Dignam R, Czaja SJ, Ryvicker M, et al. Race, Ethnicity, and Other Risks for Live Discharge Among Hospice Patients with Dementia. J Am Geriatr Soc. 2020;68:551-8.

43. Russell D, Luth EA, Ryvicker M, Bowles KH, Prigerson HG. Live Discharge From Hospice Due to Acute Hospitalization: The Role of Neighborhood Socioeconomic Characteristics and Race/ Ethnicity. Med Care. 2020;58:320-8.

44. Gardner DS, Doherty M, Bates G, Koplow A, Johnson S. Racial and Ethnic Disparities in Palliative Care: A Systematic Scoping Review. Fam Soc. 2018;99:301-16.

45. Elk R, Emanuel L, Hauser J, Bakitas M, Levkoff S. Developing and Testing the Feasibility of a Culturally Based Tele-Palliative Care Consult Based on the Cultural Values and Preferences of Southern, Rural African American and White Community Members: A Program by and for the Community. Health Equity. 2020;4:52-83.

46. Kagawa-Singer M, Blackhall LJ. Negotiating cross-cultural issues at the end of life: "You got to go where he lives." JAMA. 2001;286:2993-3001.

47. Cain CL, Surbone A, Elk R, Kagawa-Singer M. Culture and Palliative Care: Preferences, Communication, Meaning, and Mutual Decision Making. J Pain Symptom Manage. 2018;55:1408-19.

48. Searight HR, Gafford J. Cultural diversity at the end of life: issues and guidelines for family physicians. Am Fam Physician. 2005;71:515-22.

49. Ersek M, Kagawa-Singer M, Barnes D, Blackhall L, Koenig BA. Multicultural considerations in the use of advance directives. Oncol Nurs Forum. 1998;25:1683-90.

50. Krauker EL, et al. Barriers to Optimum End-of-life Care for Minority Patients. 2002. https://agsjournals.onlinelibrary.wiley. com/doi/abs/10.1046/j.1532-5415.2002.50027.x. Cited 26 Jun 2021
51. Mitchell BL, Mitchell LC. Review of the literature on cultural competence and end-of-life treatment decisions: the role of the hospitalist. J Natl Med Assoc. 2009;101:920-6.

52. Quill TE, Abernethy AP. Generalist plus Specialist Palliative Care - Creating a More Sustainable Model. 2013. https://www. nejm.org/doi/10.1056/NEJMp1215620. Cited 21 Jul 2021

53. •Watts KA, Gazaway S, Malone E, Elk R, Tucker R, McCammon S, et al. Community Tele-pal: A community-developed, culturally based palliative care tele-consult randomized controlled trial for African American and White Rural southern elders with a life-limiting illness. Trials. 2020;21:672.

54. Hart AS, Matthews AK. End-of-Life Interventions for African Americans With Serious Illness: A Scoping Review. J Hosp Palliat Nurs. 2021;23:9-19.

55. Mazanec PM, Daly BJ, Townsend A. Hospice Utilization and End-of-Life Care Decision Making of African Americans. Am J Hosp Palliat Med. 2010;27:560-6.

56. Bullock K. The Influence of Culture on End-of-Life Decision Making. J Soc Work End Life Palliat Care. 2011;7:83-98.

57. Gross TT, Story CR, Harvey IS, Allsopp M, Whitt-Glover M. "As a Community, We Need to be More Health Conscious": Pastors' Perceptions on the Health Status of the Black Church and African-American Communities. J Racial Ethn Health Disparities. 2018;5:570-9.

58. Cardona M, Lewis E, Shanmugam S, Nicholson M, Williamson M, Hanly L, et al. Dissonance on perceptions of end-of-life needs between health-care providers and members of the public: Quantitative cross-sectional surveys. Australas J Ageing. 2019;38:e75-84.

59. Romo RD, Allison TA, Smith AK, Wallhagen MI. Sense of Control in End-of-Life Decision-Making. J Am Geriatr Soc. 2017;65:e70-5.

60. $\bullet$ Peck V, Valiani S, Tanuseputro P, Mulpuru S, Kyeremanteng K, Fitzgibbon E, et al. Advance care planning after hospital discharge: qualitative analysis of facilitators and barriers from patient interviews. BMC Palliat Care. 2018;17:127.

61. Balaban RB. A Physician's Guide to Talking About End-of-Life Care. J Gen Intern Med. 2000;15:195-200.

62. Bernacki RE, Block SD. Communication About Serious Illness Care Goals: A Review and Synthesis of Best Practices. JAMA Intern Med. 2014;174:1994-2003.

63. Carr D, Luth EA. Advance Care Planning: Contemporary Issues and Future Directions. 2017. https://academic.oup. com/innovateage/article/1/1/igx012/4096494. Cited 28 May 2020

64. Periyakoil VS, O’Mahony S, Elk R, Quill T. Our Differences Make Us Stronger. J Pain Symptom Manage. 2018;55:1398-9.

65. Winter L. Patient Values and Preferences for End-of-Life Treatments: Are Values Better Predictors Than a Living Will? J Palliat Med. 2013;16:362-8.

66. Hawkins NA, Ditto PH, Danks JH, Smucker WD. Micromanaging death: process preferences, values, and goals in end-of-life medical decision making. Gerontologist. 2005;45:107-17.

67. Simon J, Porterfield P, Bouchal SR, Heyland D. "Not yet" and "Just ask": barriers and facilitators to advance care planninga qualitative descriptive study of the perspectives of seriously ill, older patients and their families. BMJ Support Palliat Care. 2015;5:54-62.

68. Douglas Pamela S. Williams Kim Allan, Walsh Mary Norine. Diversity Matters J Am Coll Cardiol. 2017;70:1525-9.

69. Mayo AT, Woolley AW. Teamwork in Health Care: Maximizing Collective Intelligence via Inclusive Collaboration and Open Communication. AMA J Ethics. 2016;18:933-40.

70. Gausvik C, Lautar A, Miller L, Pallerla H, Schlaudecker J. Structured nursing communication on interdisciplinary acute care teams improves perceptions of safety, efficiency, understanding 
of care plan and teamwork as well as job satisfaction. J Multidiscip Healthc. 2015;8:33-7.

71. $\bullet P f e i f e r$ M, Head BA. Which Critical Communication Skills Are Essential for Interdisciplinary End-of-Life Discussions? AMA J Ethics. 2018;20:724-31.

72. Elk R, Barnett M, Thompson M, Tate V, Nichols A. African American Communities Speak to Palliative Care Clinicians: Evaluation of an Innovative Community-Developed Communication Skills Training Program (GP726). J Pain Symptom Manage. 2020;60:260-1.

73. Cuevas AG, O'Brien K. Racial centrality may be linked to mistrust in healthcare institutions for African Americans. J Health Psychol. 2019;24:2022-30.

74. Boucher NA, Johnson KS. Cultivating Cultural Competence: How Are Hospice Staff Being Educated to Engage Racially and Ethnically Diverse Patients? Am J Hosp Palliat Care. 2021;38:169-74.

75. NEJM. Stolen Breaths. 2020. https://www.nejm.org/doi/full/10. 1056/nejmp2021072. Cited 12 Jul 2021

76. Hardeman RR, Burgess D, Murphy K, Satin DJ, Nielsen J, Potter TM, et al. Developing a Medical School Curriculum on Racism: Multidisciplinary, Multiracial Conversations Informed by Public Health Critical Race Praxis (PHCRP). Ethn Dis. 2018;28:271-8.
77. Schiavo R. What is true community engagement and why it matters (now more than ever). J Commun Healthc. 2021;14:91-2.

78. Devia C, Baker EA, Sanchez-Youngman S, Barnidge E, Golub M, Motton F, et al. Advancing system and policy changes for social and racial justice: comparing a Rural and Urban Community-Based Participatory Research Partnership in the US. Int J Equity Health. 2017;16:17.

79. LaVeaux D, Christopher S. Contextualizing CBPR: Key Principles of CBPR meet the Indigenous research context. Pimatisiwin. 2009;7:1.

80. Sofolahan-Oladeinde Y, Mullins CD, Baquet CR. Using community-based participatory research in patient-centered outcomes research to address health disparities in under-represented communities. J Comp Eff Res Future Med. 2015;4:515-23.

81. Elk R. The First Step Is Recognizing, Acknowledging, and Respecting the Inequity, Disrespect, and Disregard Our African American Patients Have Experienced. J Palliat Med. 2016;19:124-5.

Publisher's Note Springer Nature remains neutral with regard to jurisdictional claims in published maps and institutional affiliations. 